\title{
Felodipine in patients with chronic heart failure: discrepant haemodynamic and clinical effects
}

\author{
L B TAN, R G MURRAY, W A LITTLER \\ From the Department of Cardiovascular Medicine, University of Birmingham, East Birmingham Hospital, \\ Birmingham
}

SUMMARY Previous open studies have suggested that felodipine, a selective calcium antagonist and vasodilator, may be useful in the treatment of heart failure. A double blind placebo controlled crossover trial was therefore conducted to investigate the clinical and haemodynamic effects of felodipine in 15 patients with chronic ischaemic heart failure in New York Heart Association symptom class III. Felodipine significantly increased resting and exercise (25 W bicycle ergometry) cardiac output without producing concomitant changes in resting or exercise heart rate or right and left ventricular filling pressures. Felodipine did not significantly improve symptom scores or exercise capacity in the group as a whole. It also resulted in significant fluid retention as shown by a rise in ankle circumference, body weight, and a fall in haematocrit.

Further research is required to elucidate the mechanism that is responsible for the discrepancy between the haemodynamic and clinical effects of felodipine in patients with moderately severe heart failure.

The ability of peripheral vasodilators to improve cardiac performance in cardiac failure has been well documented. ${ }^{1-3}$ This, together with the compelling theoretical reasons that support their use, ${ }^{4-8}$ implies that vasodilators are now accepted as a viable treatment option in the management of heart failure. Some vasodilators have also been shown to improve the functional status ${ }^{9-12}$ and prognosis ${ }^{13}$ of patients with heart failure. The calcium antagonists as a class of vasodilator, however, have not been widely accepted as a treatment option in heart failure largely because of their negative inotropic effects.

Felodipine is a new calcium antagonist vasodilator with negligible negative inotropic properties. ${ }^{14}$ It has been shown in open studies to produce beneficial haemodynamic effects ${ }^{15-17}$ and improvements in the functional capacity of patients in congestive heart failure ${ }^{18}$ The aim of this study was to establish whether these preliminary results were borne out in a double blind placebo controlled crossover trial.

Requests for reprints to Dr L B Tan, Cardiac Department, John Radcliffe Hospital, Headington, Oxford OX39DU.

Accepted for publication 9 March 1987

\section{Patients and methods}

PATIENT POPULATION

We studied 15 patients (11 men and four women, mean age 61 years, range 50-69). They all had chronic heart failure in New York Heart Association functional class III and documented coronary artery disease and were in stable sinus rhythm. At entry their maximum exercise capacity was not greater than $90 \mathrm{~W}$ on a bicycle ergometer; the exercise workload started at $10 \mathrm{~W}$ and increased by $10 \mathrm{~W} / \mathrm{min}$. The mean (SEM) maximum work performed was $12.6(1.6) \mathrm{kJ}$ (equivalent to $5.9(0.4)$ minutes of exercise). Their limiting symptoms were dyspnoea, leg fatigue, or exhaustion. Mean (SEM) left ventricular ejection fraction as assessed by cross sectional echocardiography was $25(3) \%$. Throughout the study period diuretics and digoxin were the only other agents used to treat heart failure and the average (SEM) dose of frusemide taken by the patients was 83(14) $\mathrm{mg}$ per day. The doses of digoxin and diuretics were kept constant throughout the study.

STUDY DESIGN

This study was a double blind crossover comparison of the effects of felodipine and placebo after three weeks' treatment (fig 1). It consisted of a two week 


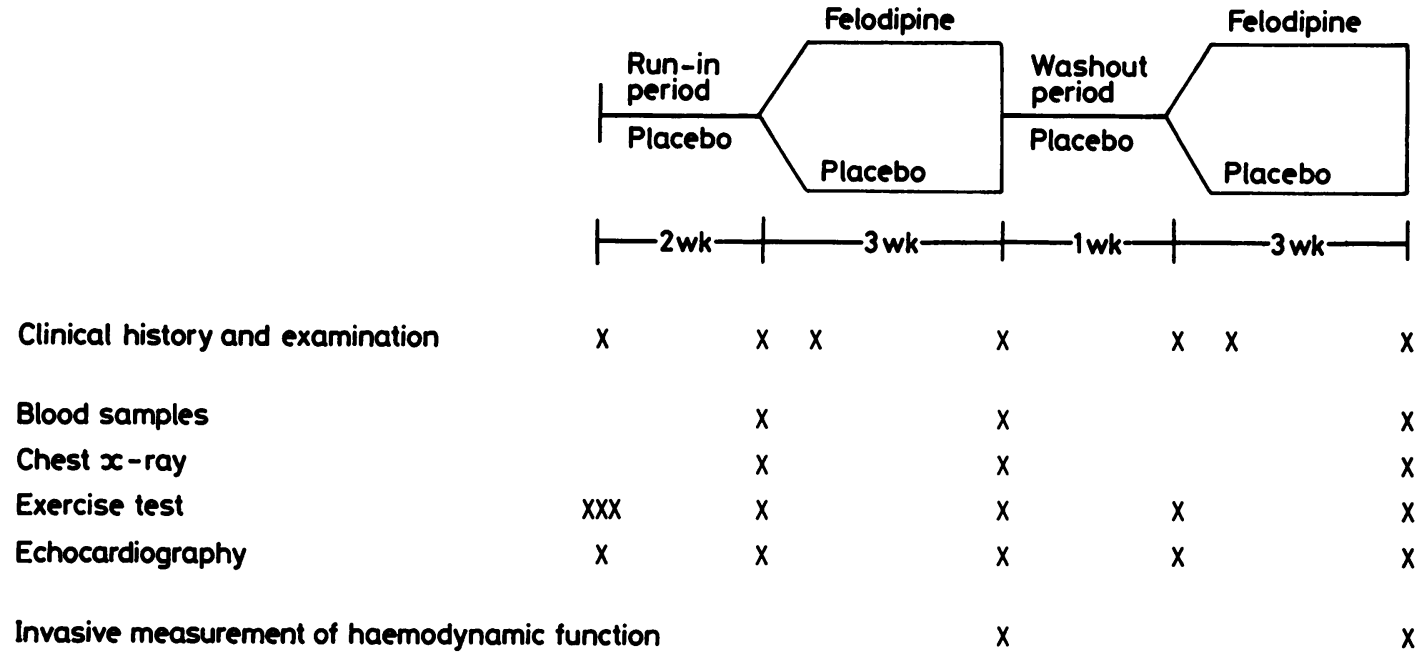

Fig 1 Study protocol.

single blind placebo run in period that started after the patient was deemed to be suitable on grounds of clinical assessments, echocardiography, and exercise testing as outlined above. At the end of this period the patient was admitted to hospital for basal clinical, echocardiographic, and exercise capacity assessments. The patient was then randomised to treatment with either felodipine or placebo. The dose aimed for was two tablets twice daily of felodipine (equivalent to $10 \mathrm{mg}$ twice a day) or placebo, but if this was not tolerated lower doses were given. After three weeks' treatment the patient was readmitted for clinical and non-invasive investigations as before, but this time invasive haemodynamic assessment was also performed. On completion of this assessment the patient was given placebo tablets for one week. At the end of this period the patient was readmitted for the same clinical and non-invasive assessment before entry into the second phase of the randomised crossover trial. Finally, at the end of this second three week period the patient was again admitted for repeat clinical and haemodynamic evaluations. The assessments were performed at the same time of day and at the same time after tablet administration in each patient. All 15 patients completed the study protocol except for one patient who refused the second right heart catheterisation.

The protocol for this study was accepted by the United Kingdom Committee on Safety of Medicines under the Clinical Trial Exemption scheme. This study was also approved by the East Birmingham Hospital Ethical Subcommittee. Informed written consent was obtained from each patient.

\section{PROCEDURES}

\section{Clinical assessments}

At each visit the patient was asked to assess progress from the previous visit using a scale of 1 to 7 as an indication of subjective clinical score: (1) markedly worse, (2) moderately worse, (3) slightly worse, (4) unchanged, (5) slightly improved, (6) moderately improved, and (7) markedly improved. The baseline clinical score was taken to be 4 , and at each subsequent visit the score increased or decreased in a cumulative fashion depending on the degree of changes from the previous visit. Any unwanted effects were recorded at the time of each clinical assessment. Ankle circumference," measured at $10 \mathrm{~cm}$ proximal to the medial maleolus, and body weight were also recorded at each visit.

\section{Exercise tests}

Maximal symptom limited exercise tests were performed on an upright bicycle ergometer by a protocol which started at $10 \mathrm{~W}$ and the workload was increased by $10 \mathrm{~W} / \mathrm{min}$. The patient's heart rate, blood pressure, and quantified symptom scores (dyspnoea, exhaustion, and/or muscle fatigue according to Borg's scale ${ }^{19}$ ) were recorded at the end of each workload.

\section{Haemodynamic measurements}

We used the electrocardiogram, thermodilution Swan-Ganz catheters, and arterial cannulas to measure the following haemodynamic variables: heart rate (HR); right atrial (RAP), pulmonary arterial (PAP), and wedge (PAWP) pressures; systemic arterial pressures (AP); and cardiac output (CO, mea- 
sured in triplicate). Baseline resting haemodynamic data were obtained at least 20 minutes after insertion of the catheters. Two control readings were taken 15 minutes apart, and the haemodynamic state was considered to be stable only if the values differed by less than $10 \%{ }^{20}$ No food or drink (except water) was given during the study period to avoid fluctuations in haemodynamic state of the patients. ${ }^{21}$ The last dose of trial medication was taken at least 12 hours before the baseline haemodynamic measurements.

The trial medication was given after recording the resting data, and the haemodynamic response was recorded 30 to 90 minutes after ingestion. The patient was then transferred to a bicycle ergometer and the haemodynamic data were collected during exercise that started at $25 \mathrm{~W}$ for three minutes and increased in steps of $10 \mathrm{~W}$ every three minutes.

\section{Data analysis}

The following haemodynamic variables were calculated: cardiac index $(\mathrm{CI}=\mathrm{CO} /$ body surface area in $\left.1 / \mathrm{min} / \mathrm{m}^{2}\right)$; stroke volume index $(\mathrm{SVI}=\mathrm{CI} / \mathrm{HR}$ in
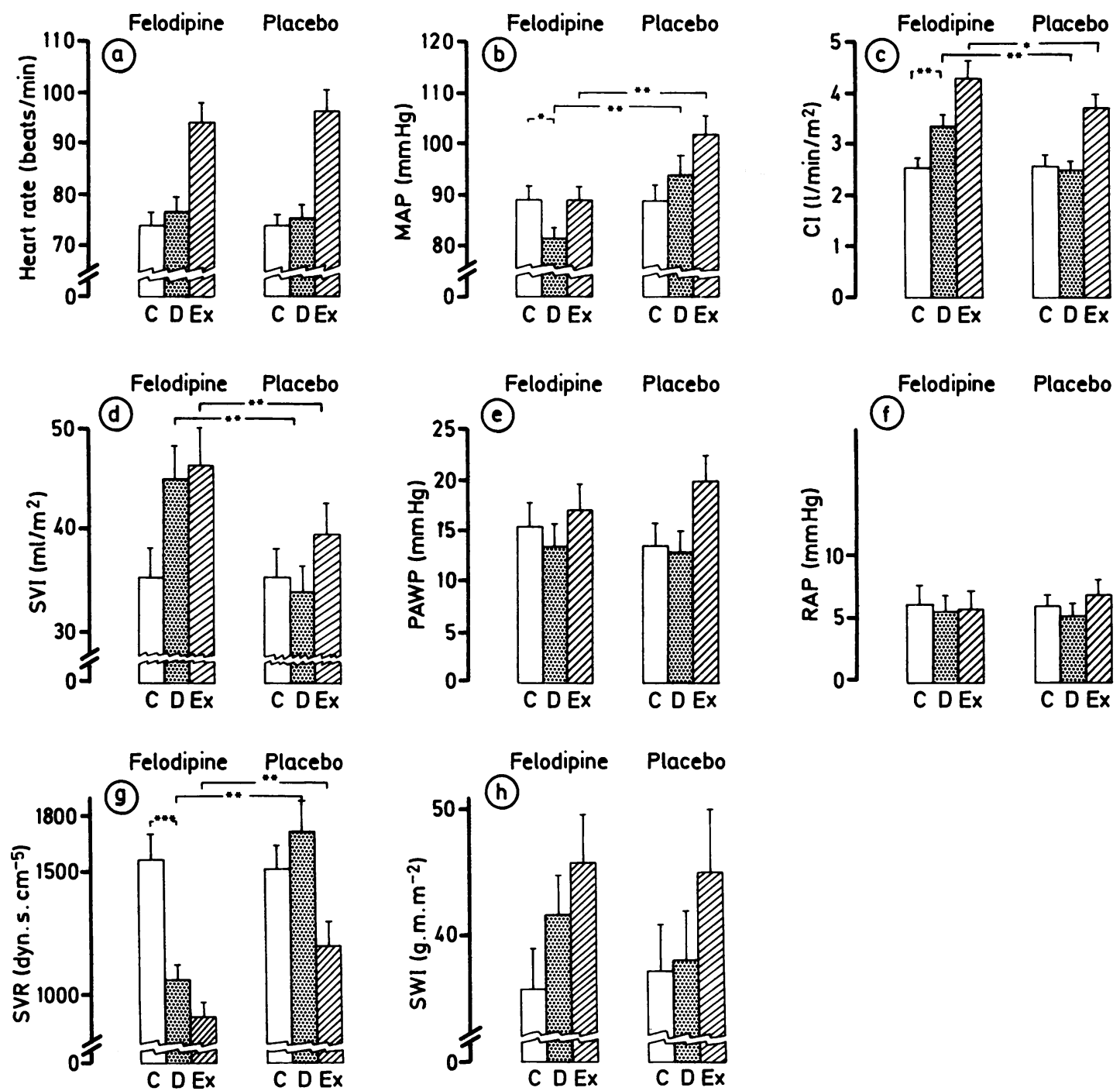

Fig 2 Haemodynamic effects of felodipine versus placebo. (a) Heart rate, (b) MAP, mean systemic arterial pressure, (c) $C I$, cardiac index, (d) SVI, stroke volume index, (e) PAWP, pulmonary artery wedge pressure, (f) $R A P$, right atrial pressure, $(g) S V R$, systemic vascular resistance, $(h) S W I$, left ventricular stroke work index. $C$, control; $D$, after drug adminstration; Ex, during $25 W$ exercise. The bars denote SEM. ${ }^{\star} p<0.05,{ }^{\star \star} p<0.01,{ }^{\star \star \star} p<0.001$. 
$\left.\mathrm{ml} / \mathrm{m}^{2}\right)$; left ventricular stroke work index (SWI = (MAP - PAWP) $\times$ SVI $\left.\times 0.0136 \mathrm{~g} \mathrm{~m} / \mathrm{m}^{2}\right)$; systemic vascular resistance $(\mathrm{SVR}=(\mathrm{MAP}-$ $\mathrm{RAP}$ )/CO $\times 80$ dyn s $\mathrm{cm}^{-5}$ ).

We used the Wilcoxon matched-pair signed ranks test for statistical analysis. Differences were taken to be significant at $p<0.05$. All results are expressed as mean (SEM).

\section{Results}

\section{RESTING AND EXERCISE HAEMODYNAMIC FUNCTION}

The haemodynamic effects of felodipine and placebo are compared in fig 2 and table 1 . The effect of felodipine on the heart rate was not significantly different from that of placebo, either at rest or during exercise at the same workload. The resting and exercise mean systemic arterial pressures were lower after felodipine than after placebo. The resting and exercise cardiac outputs were significantly greater with felodipine, and since there was no significant concomitant change in heart rate these increases were caused by felodipine induced increases in stroke volume. The pulmonary arterial wedge pressure and right atrial pressure were not significantly different after felodipine, either at rest or during exercise. The systemic vascular resistance was significantly lower after felodipine both at rest and during exercise.

CLINICAL DATA

The initial clinical score was taken to be 4 at the start of each treatment period. After the first week of treatment the score showed a tendency for symptoms to become worse on both placebo $(3.73(0.21))$ and felodipine (3.53(0.27)). After three weeks, however, the score reverted towards the control level on placebo treatment $(3.93(0.25))$, but it tended to fall further on felodipine $(3 \cdot 27(0 \cdot 40))$. This difference was not statistically significant.

The ankle circumference was significantly greater $(p<0.05)$ during treatment with felodipine $(23.0$ $(0.37) \mathrm{cm})$ than with placebo $(22.3(0.39) \mathrm{cm})$. The body weight was similarly increased $(p<0.05)$ with felodipine $(71.9(3.0) \mathrm{kg}$ vs placebo $70.9(3.1) \mathrm{kg})$.

Table 1 Effects of felodipine versus placebo

\begin{tabular}{|c|c|c|c|c|c|c|c|c|c|c|c|c|c|c|}
\hline \multirow[b]{2}{*}{ Case No } & \multirow[b]{2}{*}{ B } & \multicolumn{3}{|c|}{$P A W P$} & \multicolumn{3}{|c|}{$M A P$} & \multicolumn{3}{|l|}{$C I$} & \multicolumn{3}{|l|}{$S V I$} & \multirow{2}{*}{$\begin{array}{l}\text { Exercise* } \\
\text { duration (min) }\end{array}$} \\
\hline & & $C$ & $D$ & $E x$ & $C$ & $D$ & $E x$ & $C$ & $D$ & $E x$ & $C$ & $D$ & $E x$ & \\
\hline \multirow[t]{2}{*}{1} & $\mathbf{P}$ & 22 & 15 & 21 & 93 & 93 & 100 & $2 \cdot 8$ & $2 \cdot 8$ & 3.5 & 33 & 30 & 33 & 6 \\
\hline & $\mathbf{F}$ & 21 & 19 & 33 & 97 & 93 & 103 & $2 \cdot 7$ & $4 \cdot 1$ & $4 \cdot 8$ & 34 & 54 & 51 & 6 \\
\hline \multirow[t]{2}{*}{2} & $\mathbf{P}$ & 11 & 5 & 33 & 104 & 103 & 108 & $3 \cdot 3$ & 2.9 & $5 \cdot 4$ & 43 & 37 & 49 & 6.33 \\
\hline & $\mathbf{F}$ & 10 & 8 & 19 & 92 & 88 & 96 & $3 \cdot 1$ & $4 \cdot 1$ & 6.0 & 41 & 51 & 58 & 8 \\
\hline \multirow[t]{2}{*}{3} & $\mathbf{P}$ & 15 & 8 & 12 & 73 & 80 & 80 & $2 \cdot 4$ & 1.9 & $3 \cdot 2$ & 33 & 26 & 37 & 4 \\
\hline & F & 18 & 8 & 12 & 95 & 90 & 73 & $2 \cdot 7$ & $2 \cdot 7$ & 3.7 & 38 & 35 & 42 & $4 \cdot 5$ \\
\hline \multirow[t]{2}{*}{4} & $\mathbf{P}$ & 4 & 6 & 9 & 102 & 109 & 124 & $2 \cdot 7$ & $2 \cdot 5$ & $3 \cdot 2$ & 41 & 37 & 42 & 5 \\
\hline & $\mathbf{F}$ & 12 & 13 & 11 & 102 & 92 & 92 & $2 \cdot 8$ & 3.8 & $4 \cdot 4$ & 40 & 50 & 54 & 3 \\
\hline \multirow[t]{2}{*}{5} & $P$ & 9 & 11 & 21 & 94 & 89 & 110 & $2 \cdot 6$ & 2.5 & $5 \cdot 1$ & 38 & 35 & 64 & 9 \\
\hline & $\mathrm{F}$ & 12 & 11 & 8 & 88 & 75 & 78 & $2 \cdot 7$ & $2 \cdot 8$ & 3.5 & 49 & 52 & 58 & 6 \\
\hline \multirow[t]{2}{*}{6} & $\mathbf{P}$ & 24 & 20 & 30 & 94 & 100 & 107 & 2.5 & $2 \cdot 6$ & $3 \cdot 2$ & 37 & 34 & 34 & 6 \\
\hline & F & 32 & 23 & 24 & 89 & 72 & 90 & $2 \cdot 4$ & $3 \cdot 3$ & 4.0 & 33 & 47 & 41 & 6 \\
\hline \multirow[t]{2}{*}{7} & $\mathrm{P}$ & 6 & 7 & 9 & 97 & 132 & 124 & 2.9 & $2 \cdot 2$ & 3.8 & 45 & 37 & 40 & 4 \\
\hline & $\mathbf{F}$ & 7 & 4 & - & 109 & 83 & - & $2 \cdot 2$ & $3 \cdot 3$ & - & 33 & 47 & - & 4 \\
\hline \multirow[t]{2}{*}{8} & $\mathbf{P}$ & 30 & 28 & 31 & 96 & 96 & 114 & $1 \cdot 6$ & $2 \cdot 0$ & $2 \cdot 6$ & 25 & 29 & 33 & 7 \\
\hline & F & 32 & 28 & 31 & 98 & 89 & 101 & 1.6 & $2 \cdot 4$ & $2 \cdot 7$ & 24 & 35 & 35 & 5 \\
\hline \multirow[t]{2}{*}{9} & $\mathbf{P}$ & 15 & 18 & 17 & 83 & 93 & 96 & $4 \cdot 7$ & $4 \cdot 7$ & $6 \cdot 1$ & 65 & 65 & 66 & 7 \\
\hline & $\mathrm{F}$ & 16 & 14 & 12 & 82 & 71 & 82 & $4 \cdot 8$ & $5 \cdot 6$ & $7 \cdot 4$ & 63 & 75 & 76 & 7 \\
\hline \multirow[t]{2}{*}{10} & $\mathbf{P}$ & 8 & 6 & 13 & 79 & 80 & 98 & $1 \cdot 8$ & $2 \cdot 4$ & 3.4 & 27 & 35 & 37 & 9 \\
\hline & $\mathbf{F}$ & (refu & ed repe & right & eart cat & neterisa & tion) & & & & & & & $9 \cdot 33$ \\
\hline \multirow[t]{2}{*}{11} & $\mathbf{P}$ & 9 & 11 & 14 & 100 & 99 & 98 & 2.4 & $2 \cdot 4$ & 3.7 & 34 & 36 & 44 & 6 \\
\hline & $\mathbf{F}$ & 6 & 7 & 15 & 82 & 76 & 103 & $2 \cdot 0$ & 3.5 & $4 \cdot 3$ & 32 & 50 & 46 & $6 \cdot 5$ \\
\hline \multirow[t]{2}{*}{12} & $\mathbf{P}$ & 5 & 6 & 7 & 94 & 98 & 97 & $2 \cdot 8$ & $2 \cdot 6$ & $3 \cdot 2$ & 33 & 30 & 30 & 7 \\
\hline & $\mathbf{F}$ & 2 & 5 & 4 & 80 & 75 & 87 & $2 \cdot 2$ & $3 \cdot 6$ & $3 \cdot 2$ & 26 & 39 & 31 & $8 \cdot 17$ \\
\hline \multirow[t]{2}{*}{13} & $\mathbf{P}$ & 12 & 10 & 8 & 81 & 84 & 86 & $2 \cdot 0$ & $2 \cdot 0$ & $3 \cdot 1$ & 25 & 25 & 32 & 8 \\
\hline & $\mathbf{F}$ & 8 & 6 & 8 & 83 & 77 & 83 & $2 \cdot 0$ & $2 \cdot 8$ & 3.7 & 24 & 28 & 37 & $7 \cdot 17$ \\
\hline \multirow[t]{2}{*}{14} & $\mathbf{P}$ & 8 & 9 & 19 & 80 & 81 & 108 & $2 \cdot 4$ & $2 \cdot 5$ & 3.9 & 32 & 33 & 28 & 7 \\
\hline & $\mathbf{F}$ & 12 & 12 & 14 & 79 & 83 & 89 & $2 \cdot 7$ & 3.0 & 5.5 & 38 & 41 & 48 & 7 \\
\hline \multirow[t]{2}{*}{15} & $\mathbf{P}$ & 26 & 32 & 33 & 66 & 73 & 83 & $2 \cdot 0$ & 1.8 & 2.5 & 22 & 20 & 25 & 9 \\
\hline & $\mathbf{F}$ & 27 & 31 & 32 & 73 & 79 & 83 & $2 \cdot 0$ & $2 \cdot 4$ & 2.9 & 21 & 26 & 27 & 9 \\
\hline Mean & $\mathrm{P}$ & 13.6 & $12 \cdot 8$ & $19 \cdot 8$ & $89 \cdot 1$ & 94.0 & $102 \cdot 2$ & $2 \cdot 6$ & $2 \cdot 5$ & 3.7 & 35.4 & $33 \cdot 8$ & $39 \cdot 6$ & $6 \cdot 7$ \\
\hline (SEM) & & $2 \cdot 1$ & $2 \cdot 1$ & $2 \cdot 8$ & 2.9 & 3.8 & $3 \cdot 4$ & $0 \cdot 2$ & 0.2 & 0.3 & $2 \cdot 7$ & $2 \cdot 6$ & $3 \cdot 1$ & 0.4 \\
\hline Mean & $\mathbf{F}$ & $15 \cdot 4$ & 13.5 & $17 \cdot 1$ & $89 \cdot 2$ & 81.6 & $89 \cdot 2$ & $2 \cdot 6$ & $3 \cdot 4$ & $4 \cdot 3$ & $35 \cdot 3$ & 44.9 & $46 \cdot 3$ & $6 \cdot 4$ \\
\hline (SEM) & & 2.5 & $2 \cdot 2$ & 2.5 & 2.6 & 2.0 & $2 \cdot \overline{5}$ & $0 \cdot 2$ & 0.2 & 0.4 & 3.0 & $3 \cdot 3$ & $3 \cdot 7$ & 0.5 \\
\hline
\end{tabular}

C, control at rest; CI, cardiac index; D, after drug administration at rest; F, felodipine; MAP, mean arterial pressure; P, placebo: PAWP, pulmonary artery wedge pressure; SVI, stroke volume index.

$\star$ Symptom limited exercise duration without invasive monitoring. 
Table 2 Unwanted effects experienced by patients in a double blind crossover trial of felodipine and placebo

\begin{tabular}{lll}
\hline Symptoms & Felodipine & Placebo \\
\hline Headache & 8 & 5 \\
Palpitation & 3 & 2 \\
Flushing & 4 & 1 \\
Faintness/dizziness & 6 & 5 \\
Peripheral oedema & 6 & 3 \\
Increased angina & 5 & 3 \\
Tiredness & 8 & 7 \\
Sedation & 5 & 6 \\
Insomnia & 7 & 6 \\
Depression & 3 & 0 \\
Nausea & 5 & 5 \\
Heartburn & 1 & 3 \\
Indigestion & 0 & 2 \\
Flatulence & 3 & 1 \\
Constipation & 5 & 3 \\
Rash & 0 & 1 \\
Increased dyspnoea & 6 & 7 \\
Wheezing & 1 & 2 \\
\hline
\end{tabular}

Table 2 shows the unwanted effects reported by the patients. Apart from symptoms related to vasodilation, felodipine did not appear to cause any more unwanted effects than placebo. Three patients had to have the dose of the study drug reduced because of unwanted effects when they were taking felodipine. None of the patients on placebo needed a reduction in the dose.

\section{EXERCISE TOLERANCE TESTS}

On entry into the study 12 patients had dyspnoea, one had leg fatigue, and in two both symptoms limited exercise. During the double blind study periods
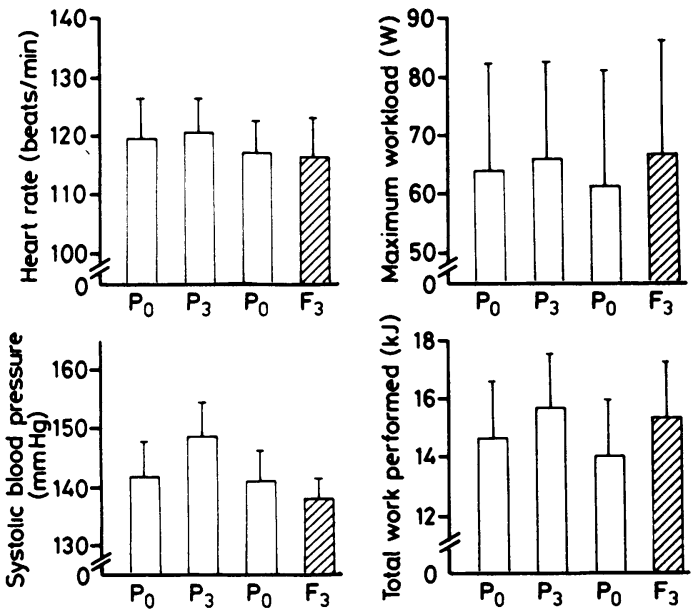

Fig 3 Maximal exercise test results for patients during treatment with felodipine or placebo: maximum heart rate, and systolic blood pressure at end of exercise, maximum workload, total work performed. Bars denote SEM. $P_{0}$, at the beginning of either treatment period; $P_{3}$, after three weeks of placebo; and $F_{3}$, after three weeks of felodipine. there was a tendency for more patients to have leg fatigue as a limiting symptom on felodipine (nine patients) than on placebo at different stages of the protocol (5-6 patients). There was no difference in the frequency of dyspnoea (10-11 patients on each treatment).

Figure 3 shows the workload achieved and the heart rate and blood pressure at the end of symptom limited exercise. There was no significant difference in the highest workload achieved and the total work performed between patients on felodipine and on placebo. The heart rate and blood pressure at the end of exercise were also similar on felodipine and placebo. The exercise capacity at various stages during the trial when the patients were taking placebo showed no significant training effect.

HAEMATOLOGICAL AND BIOCHEMICAL RESULTS Most standard haematological or biochemical indices did not change significantly during either of the treatment periods; however, serum potassium (3.9 (0.12) vs $4.2(0.12) \mathrm{mmol} / \mathrm{l}, \mathrm{p}<0.05)$, the haematocrit $(41.5(0.9) \%$ vs $43.5(0.8) \%, \mathrm{p}<0.05)$, and the erythrocyte count $(4.59(0.13) 4.81(0.11) \times$ $\left.10^{12} / 1, \mathrm{p}<0.05\right)$ fell while patients were taking felodipine. The results shown are those at the end of three weeks on felodipine and at the end of three weeks on placebo.

\section{CHEST RADIOGRAPHY}

The cardiothoracic ratio and the size of the cardiac silhouette did not change during either of the treatment periods.

\section{Discussion}

Unlike previous studies which suggested that felodipine can produce favourable effects in heart failure $^{15-18}$ our study did not show that this agent improved the symptoms and exercise capacity of a group of patients with chronic heart failure, despite its ability to produce desirable haemodynamic effects (as reflected by the increased cardiac index at rest and during exercise). Felodipine had considerable undesirable effects in our patients. Ankle circumference, body weight, and the fall in haematocrit showed that they retained more fluid during treatment with felodipine than with placebo.

When Emanuelsson et al studied the short term haemodynamic effects of felodipine they reported a significant decrease in heart rate and pulmonary capillary wedge pressure from baseline to postfelodipine period. ${ }^{15}$ In a similar group of patients Timmis et al found no decrease in heart rate, but there was a large fall in pulmonary wedge pressure. ${ }^{16}$ When we used comparable doses of felo- 
dipine (5-10 mg) in a double blind study we found no significant change in either heart rate or pulmonary artery wedge pressure between baseline and post-felodipine values and between placebo and felodipine treatment. It is impossible to state whether these differences were caused by dissimilarity in patient populations studied or observer bias.

The disparity between the results obtained in this double blind study and those obtained previously in open studies underlines the importance of controlled randomised trials. This is particularly true when obtaining subjective data, such as symptoms and exercise capacity. In another open study, Timmis and colleagues reported that the exercise capacity of their patients with heart failure was improved by felodipine, ${ }^{18}$ but this was not confirmed by the results of our double blind study. They attributed the felodipine induced increase in exercise capacity to the attenuation of the rise in left ventricular filling pressure during exercise. However, when we identified the subgroup of patients whose left ventricular filling pressure during submaximal exercise $(25 \mathrm{~W})$ was lower with felodipine $(16.4$ (3.64) $\mathrm{mm} \mathrm{Hg}$ vs $25 \cdot 7$ (4.23) $\mathrm{mm} \mathrm{Hg}$ with placebo) we found that felodipine did not increase their exercise capacity $(18.56(2.03) \mathrm{kJ}$ on felodipine vs $18 \cdot 13(2.09) \mathrm{kJ}$ on placebo). Our result is consistent with the findings of other investigators who studied other arteriolar dilators, for example hydralazine and minoxidil, in randomised studies ${ }^{223}$ where beneficial effects on haemodynamic criteria were not translated to clinical improvements.

Why did the haemodynamic findings appear to be discrepant with the clinical outcome and results of exercise test? There are several possible explanations. The study may not have been long enough. However, the unwanted effects of felodipine in certain patients, such as fluid retention, might have become worse if the study had been prolonged. Furthermore, previous studies have shown that short term haemodynamic effects of vasodilators do not, in general, correlate with long term clinical response. ${ }^{24}$ Hence, the longer the treatment period, the less likely was the haemodynamic assessment to be able to predict the clinical outcome. Alternatively, the study may have been too long and tolerance may be responsible for the discrepancy. This is unlikely because the haemodynamic effects have been shown to be sustained even after a longer period of treatment. ${ }^{18}$ The dosages used may not be high enough. This is also unlikely because considerable vasodilation was observed after the administration of felodipine at the dosages given, and higher doses would be associated with worse side effects.

It may be that the wrong type of patients were studied. In conducting this study, we took great care to study a homogeneous population of patients with heart failure-they all had New York Heart Association class III heart failure caused by coronary artery disease with a left ventricular ejection fraction of $25(3) \%$ and exercise capacity of $12 \cdot 6(1 \cdot 6) \mathrm{kJ}$. But patients in heart failure are still likely to be very heterogeneous, and various factors, including valve dysfunction, rhythm or conduction abnormalities, ventricular aneurysms, inhomogeneous myocardial contraction, triggering of hormonal compensatory mechanisms, and varied response to drug treatment would contribute to the heterogeneity. The group as a whole may not show a positive clinical response to felodipine and this may be because the proportion of patients who showed beneficial effects did not exceed those who showed deterioration. Nevertheless, in this study those who had enhanced exercise capacity with felodipine had similar felodipine induced increases in cardiac output and left ventricular stroke work as those in whom exercise capacity was reduced. It may be that such drug induced increases in cardiac output and left ventricular stroke work do not necessarily lead to drug related improvements in clinical status and exercise capacity.

There is as yet no satisfactory explanation for the discrepancy between haemodynamic data and clinical findings. It is crucial to resolve this discrepancy because drug development is in part dependent on objective data obtained from haemodynamic measurements. If the results from haemodynamic evaluation cannot be related to the clinical effects of drugs, then alternative methods of assessment will have to be found, especially for the initial phases of drug development. However, before adopting this approach and rather than abandoning the practice of haemodynamic evaluation of the efficacy of vasodilator treatment, as has been suggested by Franciosa $e t a l,{ }^{25}$ we must carefully reconsider how to interpret haemodynamic data. More research is required to resolve the reported discrepancy.

We thank Mrs Elsie Gale and her team of technicians who helped to conduct this study. We thank Astra Clinical Research Unit, Edinburgh, for their generous financial support and help in data analysis, and AB Hässle, Mölndal, Sweden, for supplying the felodipine tablets.

\section{References}

1 Chatterjee K, Drew D, Parmley WW, Klausner SC, Polansky J, Zacherle B. Combination vasodilator therapy for severe chronic heart failure. Ann Intern Med 1976;85:467-70. 
2 Franciosa JA, Pierpont G, Cohn JN. Hemodynamic improvement after oral hydralazine in left ventricular failure. Ann Intern Med 1977;86:388-93.

3 Massie B, Ports T, Chatterjee K, et al. Long-term vasodilator therapy for heart failure: clinical response and its relationship to hemodynamic measurements. Circulation 1981;63:269-78.

4 Chatterjee K. Vasodilator therapy for heart failure. Ann Intern Med 1975;83:421-3.

5 Chatterjee K, Parmley WW. The role of vasodilator therapy in heart failure. Prog Cardiovasc Dis 1976;19:301-25.

6 Cohn JN, Franciosa JA. Vasodilator therapy of cardiac failure. N Engl J Med 1977;297:27-31, 254-8.

7 Braunwald E. Vasodilator therapy-a physiologic approach to the treatment of heart failure. $N$ Engl $J$ Med 1977;297:331-3.

8 Mason DT. Afterload reduction and cardiac performance. Physiologic basis of systemic vasodilators as a new approach in treatment of congestive heart failure. Am J Med 1978;65:106-25.

9 Captopril Multicenter Research Group. A placebocontrolled trial of captopril in refractory chronic heart failure. J Am Coll Cardiol 1983;2:755-63.

10 Sharpe DN, Murphy J, Coxen P, Hannan SF. Enalapril in patients with chronic heart failure: a placebo-controlled, randomized, double-blind study. Circulation 1984;70:271-8.

11 Leier CV, Huss P, Magorien RD, Unverferth DV. Improved exercise capacity and differing arterial and venous tolerance during chronic isosorbide dinitrate therapy in congestive heart failure. Circulation 1983;67:817-22.

12 Cleland JGF, Dargie HJ, Ball SG, et al. Effects of enalapril in heart failure: a double blind study of effects on exercise performance, renal function, hormones, and metabolic state. Br Heart $J$ 1985; 54:305-12.

13 Cohn JN, Archibald DG, Ziesche S, et al. Effect of vasodilator therapy on mortality in chronic congestive heart failure. Results of a Veteran Administration cooperative study. $N$ Engl J Med 1986; 314:1547-52.
14 Ljung B. Vascular selectivity of felodipine. Drugs 1985;29(suppl 2):46-58.

15 Emanuelsson H, Hjalmarson A, Holmberg S, Waagstein F. Acute haemodynamic effects of felodipine in congestive heart failure. Eur J Clin Pharmacol 1985;28:489-93.

16 Timmis AD, Campbell S, Monaghan MJ, Walker L, Jewitt DE. Acute haemodynamic and metabolic effects of felodipine in congestive heart failure. Br Heart J 1984;51:445-51.

17 Tweddel AC, Hutton I. Felodipine in ventricular dysfunction. Eur Heart $J$ 1986;7:54-60.

18 Timmis AD, Smyth P, Kenny JF, Campbell S, Jewitt DE. Effects of vasodilator treatment with felodipine on haemodynamic responses to treadmill exercise in congestive heart failure. Br Heart J 1984;52:314-20.

19 Borg G. Perceived exertion as an indicator of somatic stress. Scand J Rehabil Med 1970;2:92-8.

20 McKay CR, Nana M, Kawanishi DT, et al. Importance of internal controls, statistical methods, and side effects in short-term trials of vasodilators: a study of hydralazine kinetics in patients with aortic regurgitation. Circulation 1985;72:865-72.

21 Cornyn JW, Massie BM, Unverfeth DV, Leier CV. Haemodynamic changes after meals and placebo treatment in chronic congestive heart failure. $\mathrm{Am} \mathrm{J}$ Cardiol 1986;57:238-41.

22 Franciosa JA, Weber KT, Levine TB, et al. Hydralazine in the long-term treatment of chronic heart failure: lack of difference from placebo. $\mathrm{Am}$ Heart J 1982;104:587-94.

23 Franciosa JA, Jordan RA, Wilen MM, Leddy CL. Minoxidil in patients with chronic left heart failure: contrasting hemodynamic and clinical effects in a controlled trial. Circulation 1984;70:63-8.

24 Massie BM, Kramer BL, Topic N. Lack of relationship between the short-term hemodynamic effects of captopril and subsequent clinical response. Circulation 1984;69:1135-41.

25 Franciosa JA, Dunhman WB, Leddy CL. Hemodynamic effects of vasodilators and long-term response in heart failure. $J A m$ Coll Cardiol 1984;3:1521-30. 